\title{
Articulatory coordination by muscle-linkage during bilabial utterances
}

\author{
${\text { Takayuki } \text { Ito }^{1} \text {, Hiroaki Gomi }}^{1,2,3}$ and Masaaki Honda ${ }^{1,2}$ \\ ${ }^{1}$ CREST, JST, \\ 4-1-8, Honcho, Kawaguchi, 332-0012 Japan \\ ${ }^{2}$ NTT Communication Science Laboratories, \\ 3-1 Morinosato Wakamiya, Atsugi, 243-0198 Japan \\ ${ }^{3}$ Interdisciplinary Graduate School of Science and Engineering, Tokyo Institute of Technology, \\ 4259, Nagatsudacho, Midori, Yokohama, 226-8503 Japan
}

(Received 5 March 2003, Accepted for publication 27 March 2003 )

Keywords: Muscle stiffness, Lip-jaw model, Compensatory movement, Asymmetrical property PACS number: 43.70.Aj, 43.70.Bk [DOI: 10.1250/ast.24.391]

\section{Introduction}

Previous studies [1-4] showed that the upper lip compensatory movement against the downward perturbation to the jaw is effective in maintaining labial constriction or in attaining labial contact for the production of bilabial utterances. We measured the stiffness of the muscle-linkage between the upper lip and jaw and proposed a model in which the muscle-linkage plays key role in coordinating articulators during speech [2]. To further examine the compensatory mechanism using muscle-linkage, we here investigate the coordination change of the lip-jaw system under the upward perturbation condition, and examine the directional difference between the upward and downward perturbation. Based on the experimental observations, we propose an extended lip-jaw model, which could explain the directional difference.

\section{Experiment and results}

\subsection{EXP1: Downward perturbation}

Four subjects (all males, Japanese native speakers: A, B, $\mathrm{C}$ and D) participated in this experiment. They were instructed to say a carrier sentence "kono / $a \Phi a \Phi a /$ mitai" for each trial with the assistance of beeps. Articulatory movements [upper lip (UL), lower lip + jaw (LL+J) and jaw (J)] were measured by using a three-dimensional optical position sensor (OPTOTRAK 3020). The subject's jaw was connected to a jaw manipulandum [2], which can disturb or assist jaw movement. Note that this connection did not interfere very much with speech movements under unperturbed condition. A downward perturbation, which was stepwise in shape $(4.0 \mathrm{~N})$, disturbed the jaw movement by suddenly acting in the jaw-open direction and was triggered $0,30,60,90$, or $120 \mathrm{~ms}\left(P_{1} \sim P_{5}\right.$ : the five triangles in the bottom graph of Fig. 1) after the start time of jaw elevation during the $/ a_{1} /$ utterance. Fifty trials were randomly selected among all trials (500) as perturbed trials.

Figure 1 illustrates audio signal and articulatory movements [perturbed (solid) and control (dashed) of UL, LD (labial distance: $\mathrm{LL}+\mathrm{J}$ minus $\mathrm{UL}$ ) , $\mathrm{LL}+\mathrm{J}$ and $\mathrm{J}$, during utterance of "/aФaФa/" (subject A). The solid lines represent averaged trajectory perturbed during $/ \Phi_{1} /$ utterance $\left(P_{2}\right.$ : thick) and during $/ a_{2} /$ utterance $\left(P_{4}\right.$ : thin $)$, and the dashed line indicates the averaged control trajectory.
As shown by the solid thick line in the fourth and fifth panels, LL $+\mathrm{J}$ and $\mathrm{J}$ were suddenly shifted downward by perturbation. During $/ \Phi_{1} /$, the distance between the upper and lower lips (third panel: LD) was similar to that of the control trial, because UL also responded to the perturbation just after the response of $\mathrm{J}$ and LL $+\mathrm{J}$. Although the timing of labial closure in $/ \Phi_{2} /$ was slightly different, the temporal pattern and minimum of LD was almost the same as that of the control trial.

\subsection{EXP2: Upward perturbation}

Next, we tried to confirm the upper lip response under the opposite perturbation condition, namely, upward perturbation. The upward perturbation was realized by suddenly removing an external force continuously acting in the jaw-open direction. The speech task and the number of trials were the same as in EXP1. Two male subjects (Japanese native speakers: A, E) participated. Figure 2 shows the measured audio and displacement during utterance of "/aФaФa/" (subject A), as in EXP1. The solid lines represent averaged trajectory perturbed during $/ \Phi_{1} /$ utterance $\left(P_{3}\right.$ : thick) and during $/ a_{2} /$ utterance $\left(P_{5}\right.$ : thin), and the dashed line indicates averaged control trajectory.

As shown in fourth and fifth panels in Fig. 2, LL $+\mathrm{J}$ and $\mathrm{J}$ movements suddenly shift upward by perturbation. Consequently, the additional modulation of LD is small because of the upward compensation of the upper lip (second and third panels). This indicates that compensatory movement was induced along the perturbed direction. Moreover, the initial perturbed shift (load onset $\sim 40 \mathrm{~ms}$ ) of the upper lip in $P_{3}$ was larger than the one in $P_{5}$, indicating that the compensatory movement varied according to the speech task as shown in [2].

\section{Data analysis based on lip-jaw model}

The upper lip-jaw system can be represented as in Fig. 3(a) by modeling the upper lip as a mass, jaw as a mass, and muscles and soft tissues connecting each mass as springs. Based on this model, we previously proposed [2] an estimation method of relative stiffness $\left(k_{2}\right)$ that uses data at two different timings $\left(P_{i}, P_{j}\right)$ as follows:

$$
\frac{k_{2}\left(P_{j}\right)}{k_{2}\left(P_{i}\right)}=\frac{\delta x_{u}\left(P_{j}\right)\left(\delta x_{j}\left(P_{i}\right)-\delta x_{u}\left(P_{i}\right)\right)}{\delta x_{u}\left(P_{i}\right)\left(\delta x_{j}\left(P_{j}\right)-\delta x_{u}\left(P_{j}\right)\right)},
$$




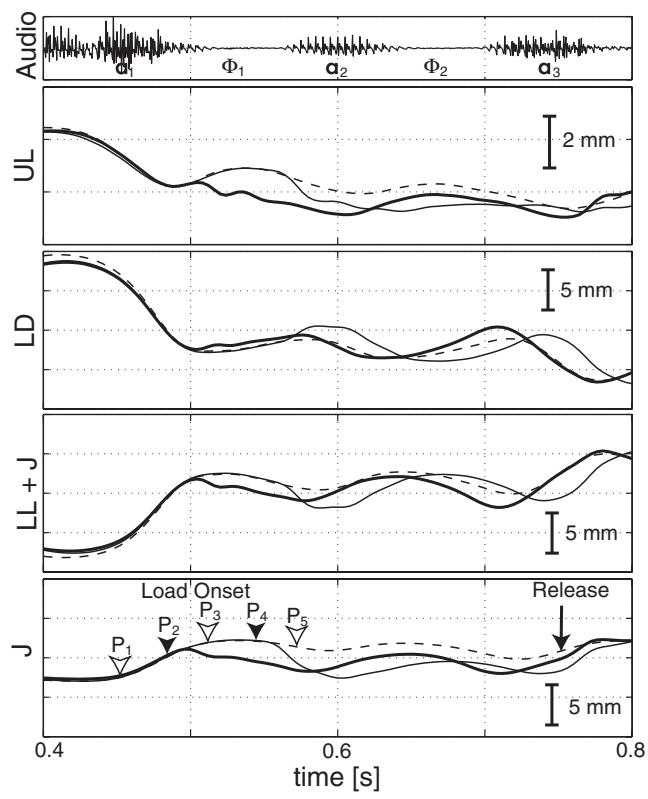

Fig. 1 Perturbed $\left(P_{2}\right.$ : thick, $P_{4}$ : thin $)$ and control (dotted) trajectory for downward perturbation.

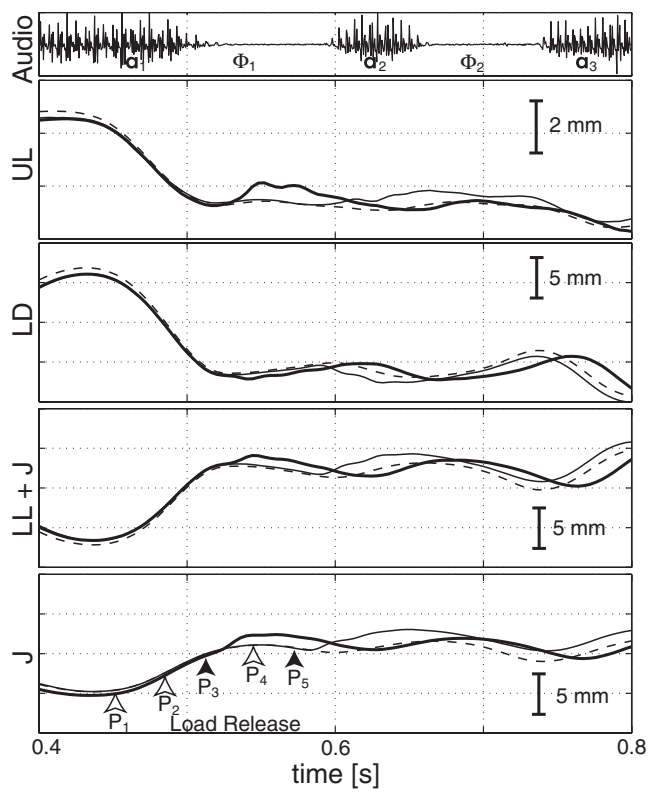

Fig. 2 Perturbed $\left(P_{3}\right.$ : thick, $P_{5}$ : thin $)$ and control (dotted) trajectory for upward perturbation.

where $\delta x_{\mathrm{u}}, \delta x_{j}$ indicate the perturbation-induced displacement, which is obtained by subtracting the control trajectory from perturbed trajectory, for the upper lip and jaw, respectively. By using this equation, we have shown that the temporal pattern of the stiffness is modulated in accordance with a speech task [2].

We here compare the stiffness between the different perturbed direction by calculating the relative stiffness, Eq. (1), from the perturbation-induced displacement for $/ \Phi_{\mathrm{d}} /$ (downward, $P_{2}$ ) and $/ \Phi_{\mathrm{u}} /$ (upward: $P_{3}$ ), and for $/ a_{\mathrm{d}} /$ (downward: $P_{4}$ ) and $/ a_{\mathrm{u}} /$ (upward, $P_{5}$ ). It appears that the stiffness associated with upward perturbation was significant-

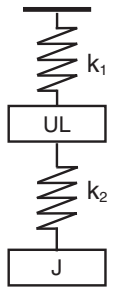

(a)UL-J model
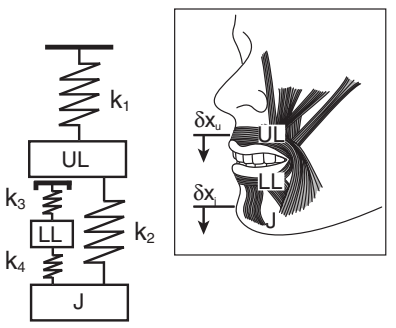

(b)UL-LL-J model
Fig. 3 Lip-jaw model $\left[k_{1}\right.$ represents the stiffness of muscles and soft tissues between the head (or upper perioral matter) and UL; $k_{2}$, between $\mathrm{UL}$ and $\mathrm{J} ; k_{3}$, between both lips; and $k_{4}$, between LL and J].

ly larger $(p<0.001)$ than the stiffness for the downward one in the $/ \Phi /$ case $\left[k_{2}\left(\Phi_{\mathrm{d}}\right) / k_{2}\left(\Phi_{\mathrm{u}}\right)=0.63 \pm 0.12\right]$ and that there was not a significant difference $(p>0.1)$ in the $/ a /$ case $\left[k_{2}\left(a_{\mathrm{d}}\right) / k_{2}\left(a_{\mathrm{u}}\right)=1.06 \pm 0.15\right]$. This suggests a directional asymmetry in perioral dynamics during / $\Phi /$ utterance and its symmetry during / $a$ / utterance.

This behavioral asymmetry during / $\Phi /$ utterance could be explained by taking into account the influence of the lower lip as follows. During / $\Phi /$, labial constriction is maintained by the contact at the lateral part of the lips near the corner of the mouth and by a coordination of the perioral muscle-linkage. In the upward perturbation, not only the muscle-linkage between the upper lip and jaw induces the upward shift of the upper lip; the lower lip also contributes. In the downward perturbation, the lateral labial contact does not largely contribute to produce a downward shift of the upper lip. The muscle and soft tissue connections between lip and jaw twitch the upper lip, causing downward movement.

According to this consideration, the upper lip-jaw system is remodeled using the stiffness $k_{3}, k_{4}$ associated with the lower lip as shown in Fig. 3(b). In this extended model, under the upward perturbed condition, not only stiffness $k_{2}$ but also stiffness $k_{3}$ and $k_{4}$ contribute to the compensatory movement by causing the lips to make contact. Under the downward perturbed condition, only stiffness $k_{2}$ contributes to produce the compensation. Because of this mechanism, the asymmetry could be generated by the different stiffness set along the perturbation direction. During $/ a /$, on the other hand, spring $k_{2}$ acts under both conditions because lateral labial contact never occurs. Thus, the stiffness values during $/ a /$ measured using the upward and downward perturbation are the same.

\section{Conclusion}

We examined compensatory movements of the upper lip induced by upward and downward jaw-perturbations, and explained the asymmetrical compensatory mechanism during a bilabial fricative consonant based on an extended lip-jaw model. These results and considerations support our hypothesis that passive properties (muscle linkage and labial contact) contribute to the coordination among articulators during speech. We thank K. Ishii and T. Hirahara (NTT CS Labs.) for their continuing encouragement. 


\section{References}

[1] J. W. Folkins and J. H. Abbs, "Lip and Jaw motor control during speech: Responses to resistive loading of the jaw," $J$. Speech Hear. Res., 18, 207-220 (1975).

[2] H. Gomi, T. Ito, E. Z. Murano and M. Honda, "Compensatory articulation during bilabial fricative production by regulating muscle stiffness," J. Phonet., 30, 261-279 (2002).
[3] J. A. S. Kelso, B. Tuller, E. Vetikiotis-Bateson and C. A. Fowler, "Functionally specific articulatory cooperation following jaw perturbations during speech: Evidence for coordinative structures," J. Exp. Phychol.: Hum. Percept. Perform., 10, 812832 (1984).

[4] S. Shaiman, "Kinematic and electromyographic responses to perturbation of the jaw," J. Acoust. Soc. Am., 86, 78-88 (1989). 